\title{
Signal Processing Based Remote Sensing Data Simulation in Radar System
}

\author{
Renxuan Hao ${ }^{1}$ and Tan Guo ${ }^{2}$ \\ ${ }^{1}$ School of Electronic Engineering, University of Electronic Science and Technology of China, Chengdu, China \\ ${ }^{2}$ College of Communication Engineering, Chongqing University, Chongqing, China
}

Correspondence should be addressed to Renxuan Hao; jszs.zl23@163.com

Received 11 April 2017; Accepted 13 June 2017; Published 25 July 2017

Academic Editor: John N. Sahalos

Copyright (C) 2017 Renxuan Hao and Tan Guo. This is an open access article distributed under the Creative Commons Attribution License, which permits unrestricted use, distribution, and reproduction in any medium, provided the original work is properly cited.

\begin{abstract}
Range cell migration has a serious impact on the precision of image formation, especially for high-resolution and large-scale imaging. To get the full resolution and high quality of the image, the range cell migration correction in the azimuth time domain must be considered. For tackling this problem, this paper presents a novel and efficient range cell migration correction method based on curve fitting and signal processing. By emulating a curve to approximate the range-compressed echo of a strong point, the range location indexes of the strong point along the azimuth direction can be obtained under the least squares criterion. The merits of the proposed method are twofold: (1) the proposed method is robust to the uncertainty of system parameters (strong tolerance) under real flights and (2) the generalization of the proposed method is better and can be easily adapted to different synthetic aperture radar (SAR) modes (e.g., monostatic and bistatic). The experimental results on real remote sensing data from both the monostatic and the bistatic SAR demonstrate the effectiveness. The regressed distance curve is completely coincident with the trajectory of strong points of the echo. Finally, the imaging focus results also validate the efficiency of the proposed method.
\end{abstract}

\section{Introduction}

Synthetic aperture radar (SAR) is an important remote sensing technique, which has attracted more and more attention since it was proposed [1]. The most significant characteristic of SAR is its ability to obtain high-resolution microwave images day and night under all weather conditions [2]. For this reason, SAR has been widely applied to many fields, such as generation of digital elevation maps, observation of volcanic activities and flood disasters, land and sea traffic monitoring, observation of vegetation growth, monitoring of ocean currents and traveling icebergs, and detection of oil spills in the ocean [3-8].

One of the most fundamental and important techniques for SAR application is the imaging algorithm [9]. By now, many efficient imaging algorithms have been put forward, such as range-Doppler (RD) algorithm [10], chirpscaling (CS) algorithm [11], wave number domain algorithm (Omega-K) [12], and back projection (BP) algorithm [13]. Although all these algorithms are effective, $\mathrm{RD}$ algorithm is the most popular algorithm since it is simple, highly efficient, and intuitive [14].

A key problem of the $\mathrm{RD}$ algorithm is the range cell migration correction (RCMC) [15]. SAR obtains low-quality images of the target due to the relative motion between the radar and the target [16]. Therefore, to obtain highresolution images, the signals from the same scatter should be situated in one range cell. Nevertheless, it is not practical in view of the platform movement relative to the target, which is called range cell migration [15]. Generally, the movement of the range cell will result in coupling between the range direction and the azimuth direction. That is why the range cell migration must be corrected before further processing in $\mathrm{RD}$ algorithms [17-19]. The basic objective of range cell migration correction (RCMC) is to adjust the received echo of the same target back to the same range cell [20]. Traditionally, range cell migration can be corrected through time-domain interpolation or frequency-domain compensation [21]. The main problems of these methods are the excessive data operation and the narrow compatibility for 
different SAR configurations. Therefore, there remains a need for an efficient method that can solve the problems.

In this paper, a curve fitting based range cell migration correction method is proposed to solve the foregoing problem. According to the Taylor expansion theorem, the ranges between the radar and targets can be expressed as polynomials for both monostatic radar and bistatic radar [22-25]. A polynomial fitting method based on the least squares principle [26] is presented to compensate the range migration. The main idea is to approximate the trajectory of a strong point in range-compressed echo by making use of a simulation of the curve, which is fast and robust for real SAR data application. With the proposed method, the operation of RCMC will become easy, flexible, and efficient.

The following parts of this paper are organized as follows. In Section 2, the basic formulation of the range cell migration and the RCMC problem is briefly presented. The basic principle of curve fitting and the proposed CF-RCMC method is described in Section 3. In Section 4, experiments on the real monostatic and bistatic SAR data for remote sensing by using the proposed CF-RCMC method are presented and analyzed. Finally, in Section 5, a concluding remark of the present work is given.

\section{Range Cell Migration}

The simplified data acquisition geometry of the monostatic SAR is illustrated in Figure 1. The radar is carried on a platform moving along a straight line at a constant velocity $V_{T}$ and a constant attitude. The instantaneous position of the sensor is $\mathbf{P}(\eta)$, where $\eta$ denotes the slow time. The slant range from target $\mathbf{P}_{t}$ to the radar at $\eta$ is denoted by $R(\eta)$. $R_{0}$ denotes the reference range, which is defined as the nearest slant range here and is perpendicular to the velocity.

With the well-known Pythagorean Theorem, the range between the target and the radar can be given by (bistatic)

$$
\begin{aligned}
R(\eta) & =R_{T}(\eta)+R_{R}(\eta) \\
& =\sqrt{{R_{0 T}}^{2}+V_{T}^{2} \eta^{2}}+\sqrt{{R_{0 R}{ }^{2}+V_{R}^{2} \eta^{2}}^{2}}
\end{aligned}
$$

where it can be approximated by the following polynomials according to the Taylor expansion theorem:

$$
\begin{aligned}
R(\eta)= & R_{T}\left(\eta_{c}\right)+R_{R}\left(\eta_{c}\right) \\
& +\left(\frac{V_{T}^{2} \eta_{c}}{R_{T}\left(\eta_{c}\right)}+\frac{V_{R}^{2} \eta_{c}}{R_{R}\left(\eta_{c}\right)}\right)\left(\eta-\eta_{c}\right) \\
& +\frac{1}{2}\left(\frac{V_{T}^{2} \cos ^{2} \theta_{\mathrm{Tr}, c}}{R_{T}\left(\eta_{c}\right)}+\frac{V_{R}{ }^{2} \cos ^{2} \theta_{\mathrm{Rr}, c}}{R_{R}\left(\eta_{c}\right)}\right)\left(\eta-\eta_{c}\right)^{2} \\
& +\cdots,
\end{aligned}
$$

where $\eta_{c}$ denotes the azimuth center time when the beam center passes through the target; $\theta_{\mathrm{Tr}, c}$ and $\theta_{\mathrm{Rr}, c}$ denote the squint angles for the transmitter and receiver with respect to $\eta_{c} ; R_{0 T}$ and $R_{0 R}$ denote the nearest distance at the initial time $t_{0} ; V_{T}$ and $V_{R}$ denote the velocity of the radar and the target;

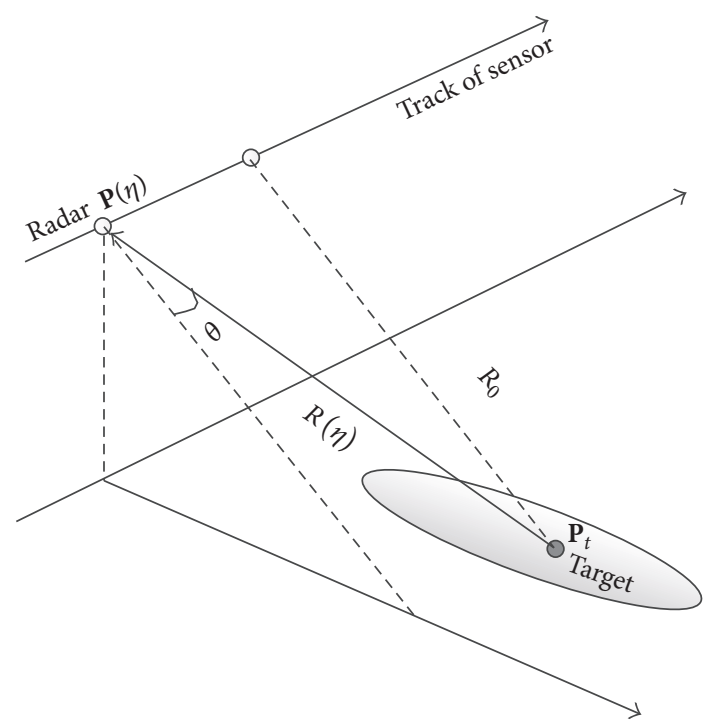

FIGURE 1: SAR geometry. The radar travels along its course at an altitude with a constant velocity $V_{T}$ and the radar transmits and receives pulses with a squint angle of $\theta$. The center of the imaging area is $P_{t}$, and the nearest slant distance between $P_{t}$ and the radar is $R_{0}$. The motion of the radar relative to the target results in RCM, which is the intrinsic feature of SAR and, however, must be corrected.

$R_{T}(\eta)$ and $R_{R}(\eta)$ denote the slant distance between the radar and the target at time $\eta$. Hence,

$$
\theta_{j r, c}=\arcsin \left(-\frac{V_{j} \eta c}{R_{j}(\eta c)}\right)
$$

where $j$ denotes the subscript $T$ or $R$.

It is not hard to realize that the range history of the monostatic SAR can be also expressed like (2). However, the expression is much more complex than that of the bistatic SAR.

Compared with the size of the range cell, the higher order terms are so small that they can be ignored:

$$
\begin{aligned}
R(\eta) & \\
= & R_{T}\left(\eta_{c}\right)+R_{R}\left(\eta_{c}\right) \\
& +\left(\frac{V_{T}^{2} \eta_{c}}{R_{T}\left(\eta_{c}\right)}+\frac{V_{R}^{2} \eta_{c}}{R_{R}\left(\eta_{c}\right)}\right)\left(\eta-\eta_{c}\right) \\
& +\frac{1}{2}\left(\frac{V_{T}^{2} \cos ^{2} \theta_{\mathrm{Tr}, c}}{R_{T}\left(\eta_{c}\right)}+\frac{V_{R}^{2} \cos ^{2} \theta_{\mathrm{Rr}, c}}{R_{R}\left(\eta_{c}\right)}\right)\left(\eta-\eta_{c}\right)^{2} .
\end{aligned}
$$

The linear and quadratic components in (4) denote the range walk and curve, respectively, and they are collectively known as range cell migration (RCM). RCM results from the motion of the radar relative to the target results. This relative motion is the base of SAR and thus RCM is the intrinsic feature of SAR. However, RCM must be corrected in the RD algorithm.

Under the condition of small squint angle, the beam center approximates the zero Doppler direction and $\eta_{c} \approx 0$, 
$\theta_{r, c} \approx 0$. So, the range history is simplified as follows (take the monostatic situation as an example):

$$
R(\eta)=R_{0}+\frac{V^{2} \eta^{2}}{2 R_{0}}
$$

where there is only range curve.

In the $\mathrm{RD}$ algorithm, the range cell migration is corrected in the range-Doppler domain. The corresponding range cell migration under the condition of side looking is [9]

$$
R\left(f_{\eta}\right)=R_{0}+\frac{\lambda^{2} R_{0} f_{\eta}^{2}}{8 V^{2}},
$$

where $f_{\eta}$ is the Doppler frequency and $\lambda$ is the wavelength.

In the range-Doppler domain, the targets with the same range have the same range cell migration and they can be corrected simultaneously, which is also the main advantage of $\mathrm{RD}$. The objective of RCMC is to remove the migrated actual track to the nearest slant range location.

\section{The Proposed Curve Fitting Based Range Cell Migration Correction (CF-RCMC) Method}

In traditional methods, RCMC is accomplished through time-domain interpolation or frequency-domain compensation. However, the main problems of these methods are the excessive data operation and the narrow compatibility for different SAR configurations.

From Figure 2, we can see that the actual track of the target is a parabola, which is in accordance with the range history in (5) or (6). If the range history curve can be known, we can use this curve to correct the range cell migration. With this idea, we apply the polynomial curve fitting to obtain this range curve through simulation.

3.1. Curve Fitting. The curve fitting method [26] is a common method that can be applied to construct the approximate expression of the function $y=f(x)$ according to a set of experimental data points $\left(x_{k}, y_{k}\right)$, where $x_{k}$ and $y_{k}$ denote the values of independent and dependent variables, respectively. Polynomial fitting is one of the most common fitting models, which can be expressed as

$$
f(x)=a_{0}+a_{1} x+a_{2} x^{2}+\cdots+a_{n} x^{n} .
$$

The fitting error between the polynomial and the measured value is

$$
e_{k}=f\left(x_{k}\right)-y_{k} \text {. }
$$

There are many kinds of criterions to minimize the fitting error. The least squares criterion is widely used and the squares error is formulated as

$$
\begin{aligned}
Q & =\sum_{k=1}^{m} e_{k}^{2}=\sum_{k=1}^{m}\left(f\left(x_{k}\right)-y_{k}\right)^{2} \\
& =\sum_{k=1}^{m}\left(a_{0}+a_{1} x_{k}+a_{2} x_{k}^{2}+\cdots+a_{n} x_{k}^{n}-y_{k}\right)^{2} .
\end{aligned}
$$

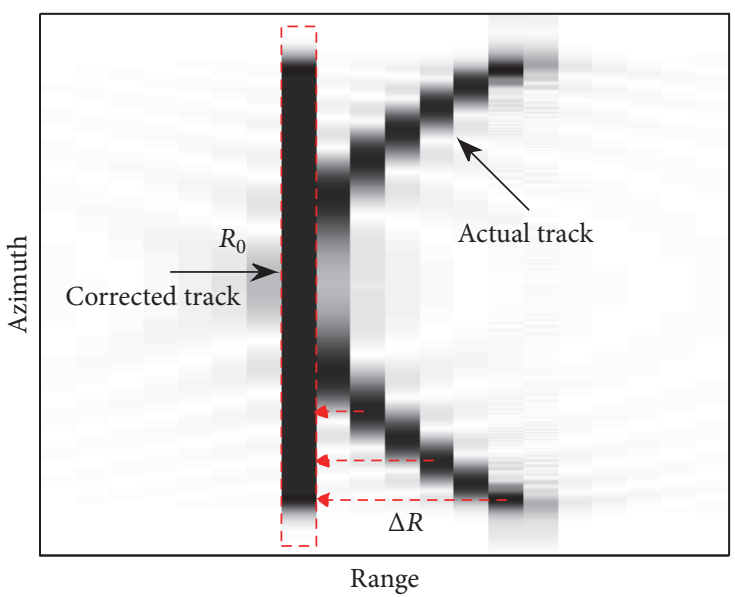

FIGURE 2: RCMC sketch. The actual curve track must be removed to the location of the nearest slant range $R_{0}$.

One can solve the equation set or utilize the optimization method by minimizing the error in (9) to obtain the fitting coefficients in (7).

3.2. The Proposed CF-RCMC. On the occasion of real data processing, the motion parameters may not be accurately obtained and the achieved curve might not fit the RCM well. In the range-compressed range-Doppler domain, the track of a strong point target can reflect RCM phenomena. However, the RCM curve of the strong point target cannot be directly obtained through the motion parameters since the strong point target is arbitrarily located. Therefore, we could adjust the motion parameters to obtain the coefficients of the quadratic polynomial. Then, we can correct the range cell migration easily with a totally known expression of the RCM curve.

The range-compressed echo is a two-dimensional data matrix with range cell migration. Figure 2 shows the range migration phenomena of a strong point target in the rangeDoppler domain. $\Delta R$ denotes the range migration of the strong point target.

The slant ranges are then transformed into range cells for data processing. In this case, the range index of a strong point target at the azimuth time $i$ is given as

$$
\operatorname{ID}(i)=\operatorname{round}\left(\frac{\left(R(i)-R_{0}\right) F_{s}}{c}\right) \text {, }
$$

where $R(i)$ is the slant range between the scene center and the radar position; $R_{0}$ is the reference range; $c$ denotes the wave propagation speed; $F_{s}$ denotes the sampling frequency. The symbol round $(\cdot)$ denotes the rounding operation.

Once all the ranges are received, they will be employed by using curve fitting under the least squares criterion. To obtain appropriate coefficients of the quadratic polynomial, we can adjust the scene center, the reference range, or the platform position, such that the simulation curve and the range migration curve of a strong point are basically coincident. Then, the obtained curve function can be used 
while 1

(1) Initialize the scene center $\mathbf{P}_{c}$ and the reference range $R_{0}$;

(2) Select $N$ azimuth times; $i=0$;

while 1

$i++$;

Compute the slant range $R(i)$ by using Equation (12);

Compute the location $\operatorname{ID}(i)$ of the strong point target in the echo by using Equation (13);

if $i>N$

break;

end if

end while

(3) Perform curve fitting on ID vector and get the simulation curve $C$.

(4) if the simulation curve $C$ coincides with the range migration line of the strong point; break;
end if

end while

(5) Remove the target echo of range cells of the nearest slant range location in frequency domain.

Pseudocode 1: Pseudocode of the proposed CF-RCMC method.

to compute the offsets. Therefore, the corresponding number of range cells where the echo should be removed can be calculated by using the simulation curve, as follows:

$$
S(i)=\operatorname{round}(f(i)-f(n))
$$

where $n$ denotes slow time of the nearest slant range.

After that, it is easy to convert the range migration line of the strong point into a line by using the properties of Fourier transformation. That is, removal in the fast time domain can be achieved through the multiplication operation in frequency domain. This operation will be done for every range cell. Finally, the RD algorithm can be used for SAR imaging.

The implementation steps of the CF-RCMC method are listed as follows.

Step 1. Initialize the scene center $\mathbf{P}_{c}$ and the reference range $R_{0}$.

Step 2. Select one azimuth time and compute the slant range between the scene center and the radar positions:

$$
R(i)=\sqrt{\left(P_{1}(i)-P_{c}\right)^{2}}+\sqrt{\left(P_{2}(i)-P_{c}\right)^{2}}
$$

where $P_{1}(i)$ and $P_{2}(i)$ denote the positions of the receiver and the transmitter, respectively. For monostatic SAR, $P_{1}(i)=$ $P_{2}(i)$.

Step 3. Compute the location of the strong point target in the echo

$$
\operatorname{ID}(i)=\operatorname{round}\left(\frac{\left(R(i)-R_{0}\right) F_{s}}{c}\right) \text {, }
$$

where the round function denotes the nearest integer operator.
Step 4. Change the azimuth times one by one, and repeat Steps 2 and 3 until the IDs at all the azimuth times are computed.

Step 5. Based on the calculated IDs, utilize the curve fitting method to obtain the quadratic polynomial coefficients.

Step 6. Adjust $P_{c}$ and $R_{0}$ and repeat Steps 1-5 until the simulation curve and range migration line of the strong point are basically coincident.

Step 7. Remove the target echo of the corresponding range cells with respect to the nearest slant range location in the range frequency domain, by making use of the properties of Fourier transformation.

Specifically, the pseudocode of the CF-RCMC method is summarized in Pseudocode 1.

Further, the flow chart of the CF-RCMC method is summarized in Figure 3.

\section{Experimental Results}

In this section, the proposed CF-RCMC method is applied to real data for both the monostatic airborne SAR and the bistatic airborne SAR. The velocities and the trajectories are not exactly known because only GPS was used to measure the antenna attitude, and hence measurement precision is not very high.

4.1. A Brief Introduction of the Radar System. The mentioned radar system in this paper is an air force military radar. The referred band is $\mathrm{x}$-band with chirp signal and the bandwidth is $300 \mathrm{MHz}$. The effective distance of the transmitter is 30 $60 \mathrm{~km}$ with a transmission power of 4000 watts (w). The effective distance of the receiver is $10 \sim 20 \mathrm{~km}$, and the data sampling frequency is $1.8 \mathrm{GHz}$. 


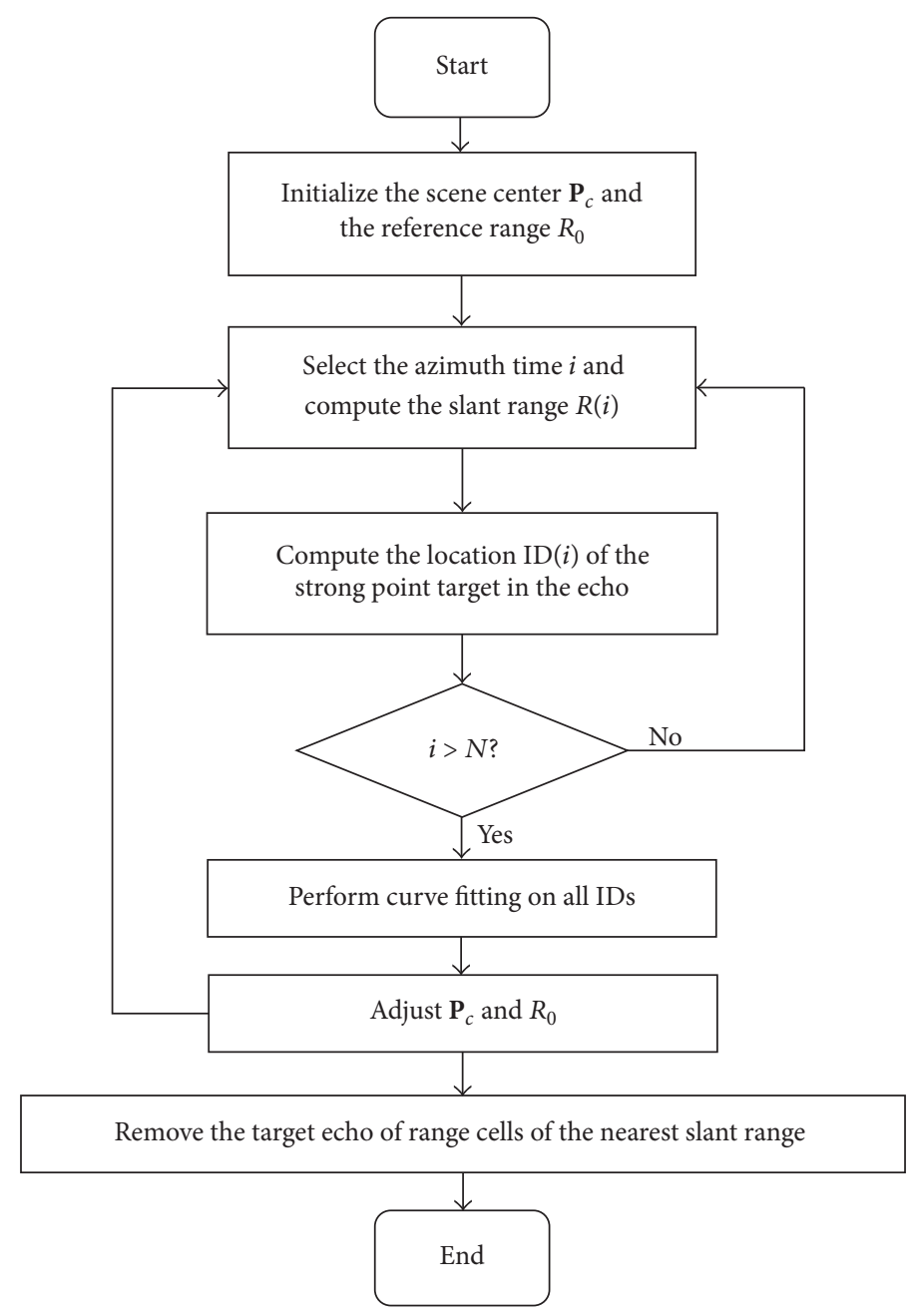

FIGURE 3: The flow chart of the proposed method, where $N$ is the total azimuth times.

4.2. Monostatic SAR Experiment. The track of a strong point target in the range-compressed echo domain is shown in Figure 4, from which we can see the range cell migration phenomenon clearly. However, the nearest slant range location does not appear in this figure. When we correct the RCM, the range at the first PRF (pulse recurrence frequency) is selected as the nearest slant range. The range cell migration is extremely serious, which is nearly 90 range cells along 5000 azimuth cells.

After adjusting the scene center and the reference range, the fitted curve (red solid line) is illustrated in Figure 5. We can see that this fitted curve is exactly coincident with the RCM curve of the strong point target. Then, we use the fitted quadratic curve expression and the properties of Fourier transformation to correct the RCM in the range frequency domain. Figure 6 shows the result of CF-RCMC. The track of the strong point target has been corrected to a horizontal straight line along the azimuth direction, which states the efficiency of the proposed CF-RCMC method. However, it is worth noting that this CF-RCMC method can only correct the RCM for partial targets around the selected one located at "the nearest slant range." In fact, all the other RCMC methods also cannot correct the RCM for every target.

After azimuth compression, the imaging results based on conventional RCMC used in $[19,20]$ and the proposed CF-RCMC are presented in Figures 7 and 8, respectively. In Figure 7 , the whole scene is seriously defocused, especially in the azimuth direction. In Figure 8, obviously, the image is well focused and the targets features can be clearly distinguished. Note that the focusing effect becomes better when the target is closer to the strong point, which is referred to correct the RCM.

4.3. Bistatic SAR Experiment. For bistatic SAR, the range history is much more complex than monostatic SAR, which thus results in more complicated RCMC. However, the proposed RCMC method based on curve fitting can be directly applied to the bistatic SAR. The processing method for bistatic SAR is totally the same as that for monostatic SAR. The results for bistatic SAR are concisely provided below.

The track of a strong point target in the range-compressed echo domain is shown in Figure 9. The range cell migration amount is about 400 range cells along 5000 azimuth cells, 


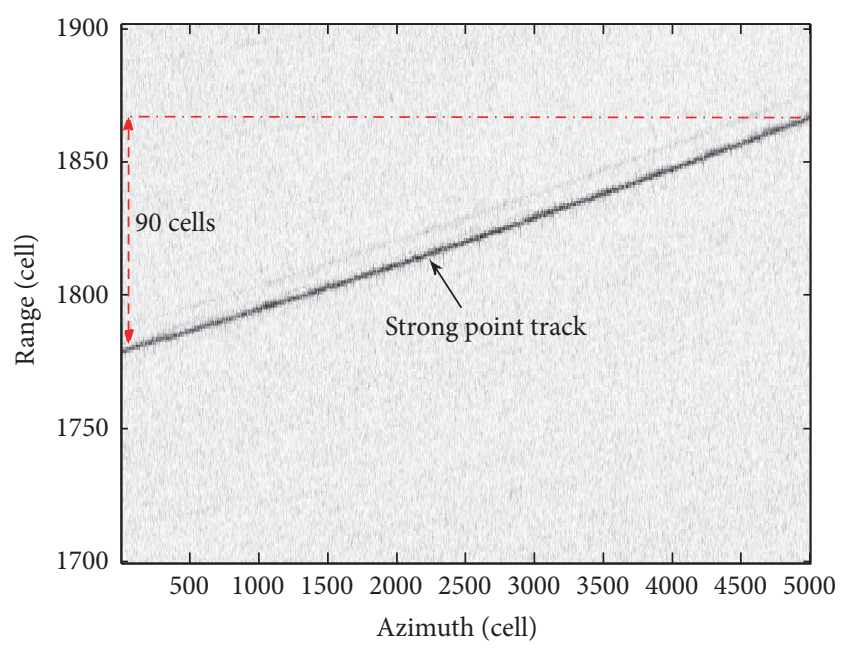

Figure 4: Track of a strong point in the range-compressed echo (monostatic SAR). The RCM of 90 cells is very serious.

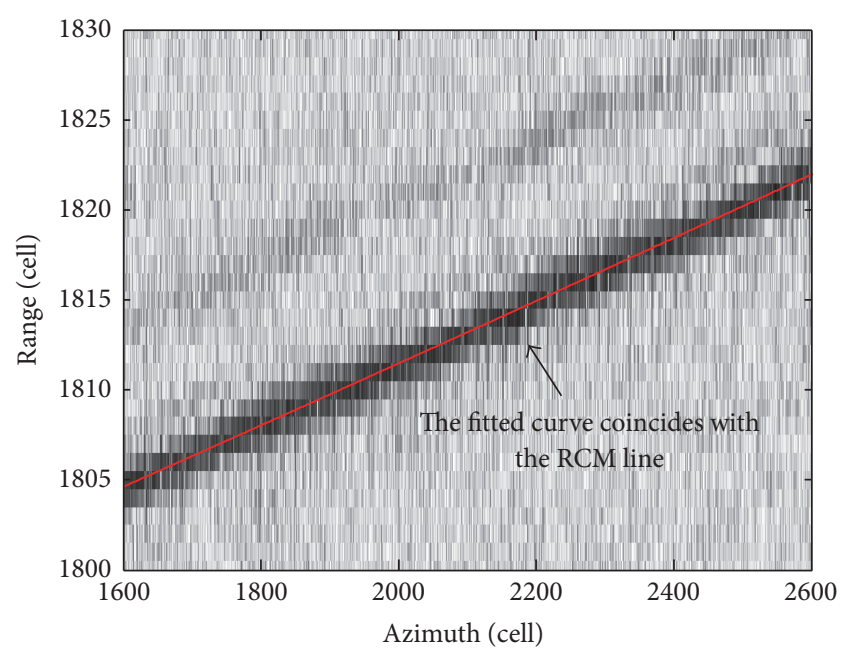

FIGURE 5: Curve fitting result (monostatic SAR). The fitted quadratic curve is coincident with the RCM line (100\% for strong points in echo), which can be used to correct the RCM.

which states that the RCM is much more serious than that in the monostatic SAR.

The fitted curve is shown in Figure 10, in which the fitted curve is exactly coincident with the RCM curve of the strong point target.

The RCMC result is illustrated in Figure 11. Without more operations than the monostatic SAR, the track of the strong point target has been corrected to a horizontal straight line along the azimuth direction. To obtain similar RCMC performance, the traditional RCMC method may not be available.

The imaging results based on the conventional RCMC method $[19,20]$ and the proposed CF-RCMC for bistatic SAR are presented in Figures 12 and 13, respectively. In Figure 12, the defocusing of the image is much more serious than that in the monostatic SAR due to the larger RCM. As seen in Figure 13, it is apparent that the CF-RCMC method

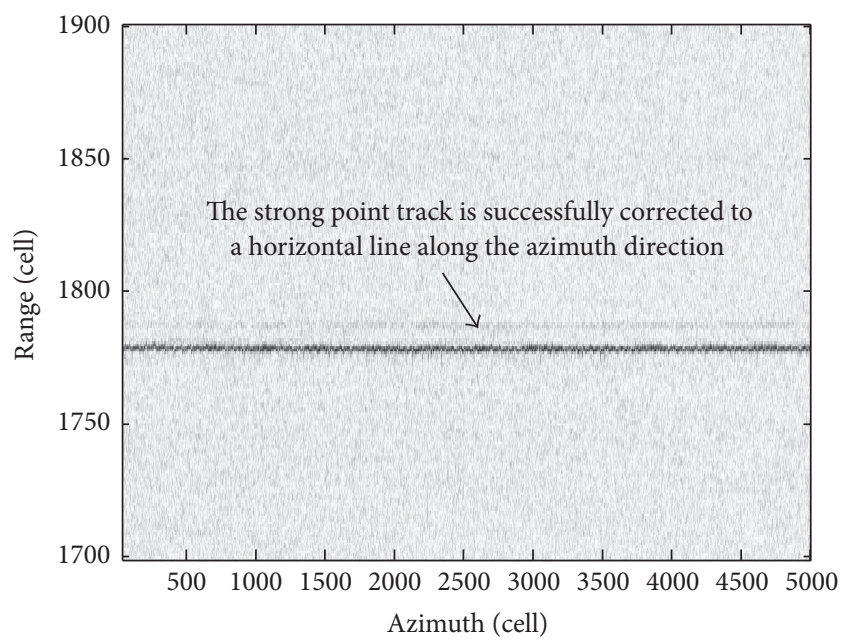

FIGURE 6: CF-RCMC result (monostatic SAR). The horizontal line indicates that the RCM has been successfully corrected.

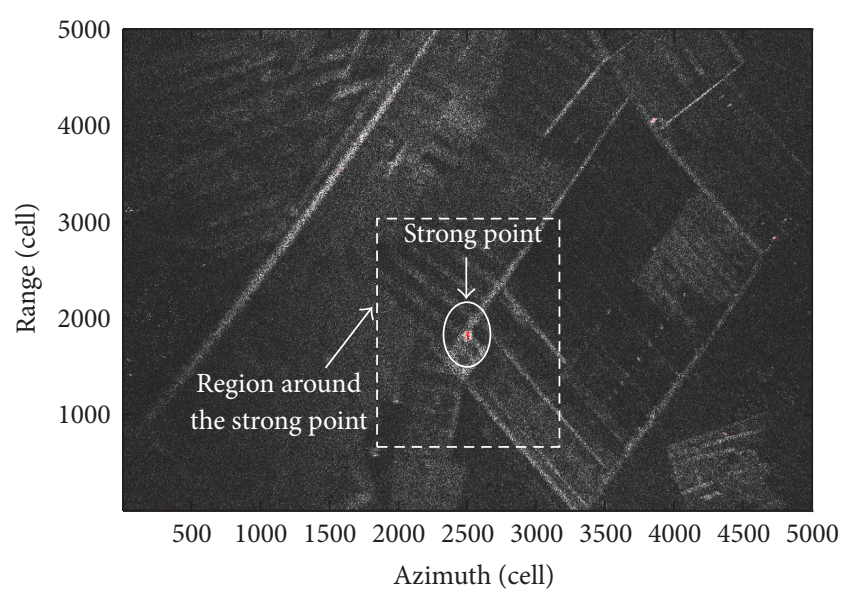

FIGURE 7: Imaging result based on conventional RCMC (monostatic SAR). The whole image is seriously defocused.

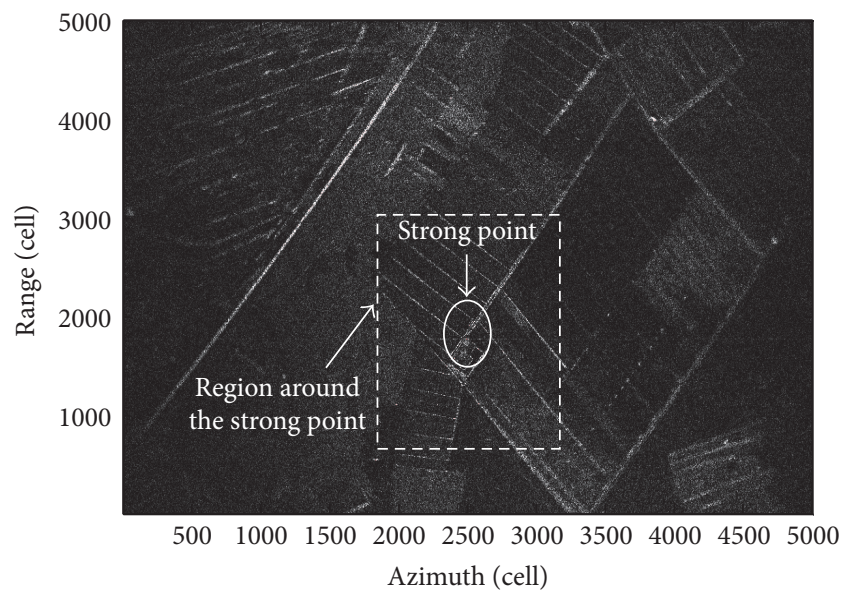

FIGURE 8: Imaging result with CF-RCMC (monostatic SAR). The image is well focused, particularly in the region around the strong point. 


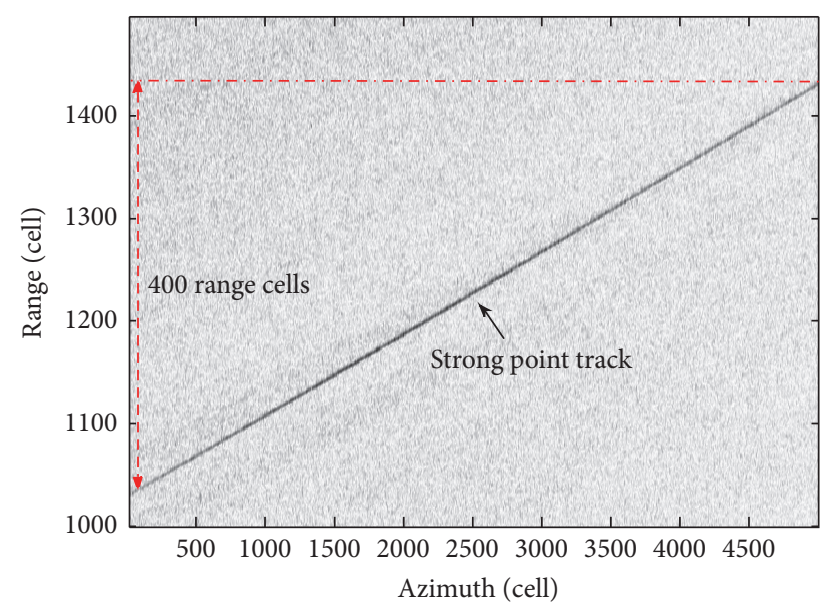

FIGURE 9: Track of a strong point in range-compressed echo (bistatic radar). The amount of RCM is much larger than that in the monostatic SAR.

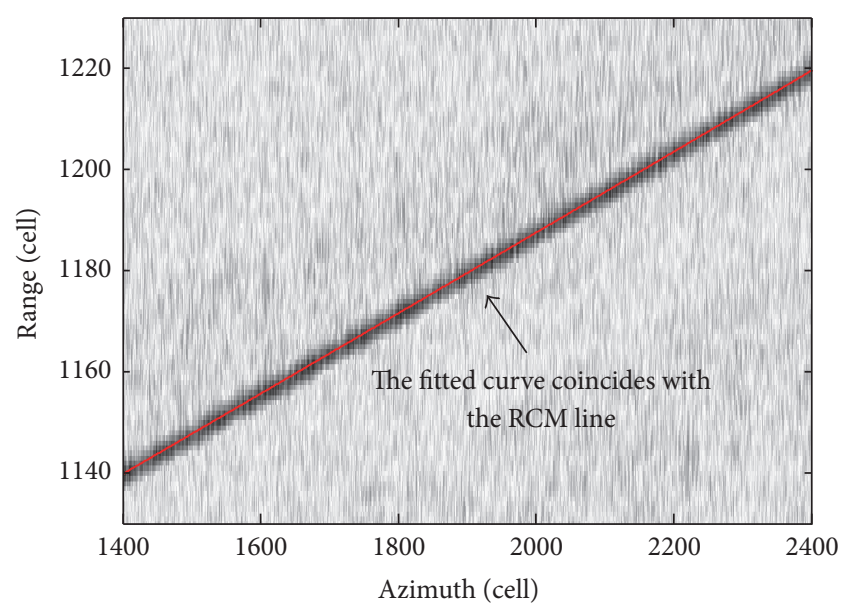

FIGURE 10: Curve fitting result (bistatic radar). The fitted curve is still coincident with the RCM line (100\% for strong points in echo).

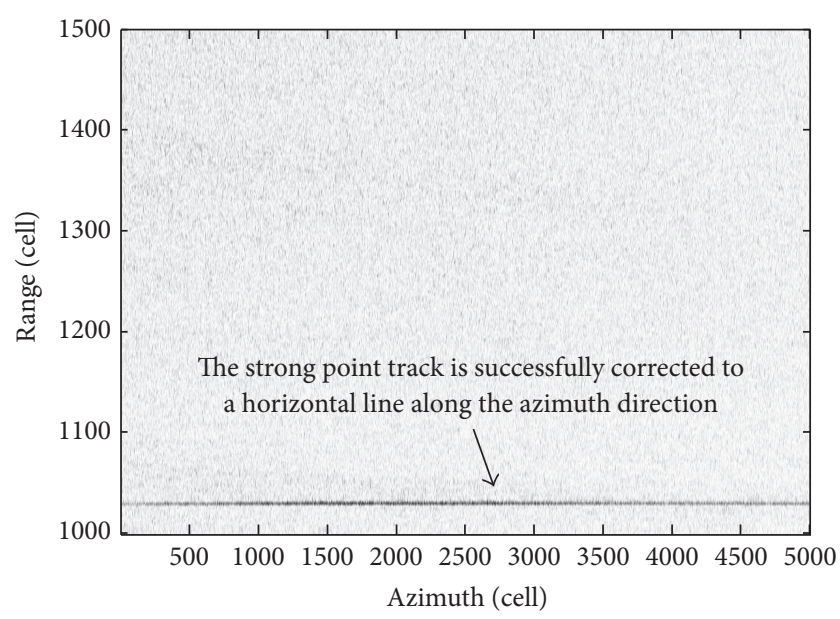

FIgURE 11: CF-RCMC result (bistatic radar). The RCM can be easily and successfully corrected.

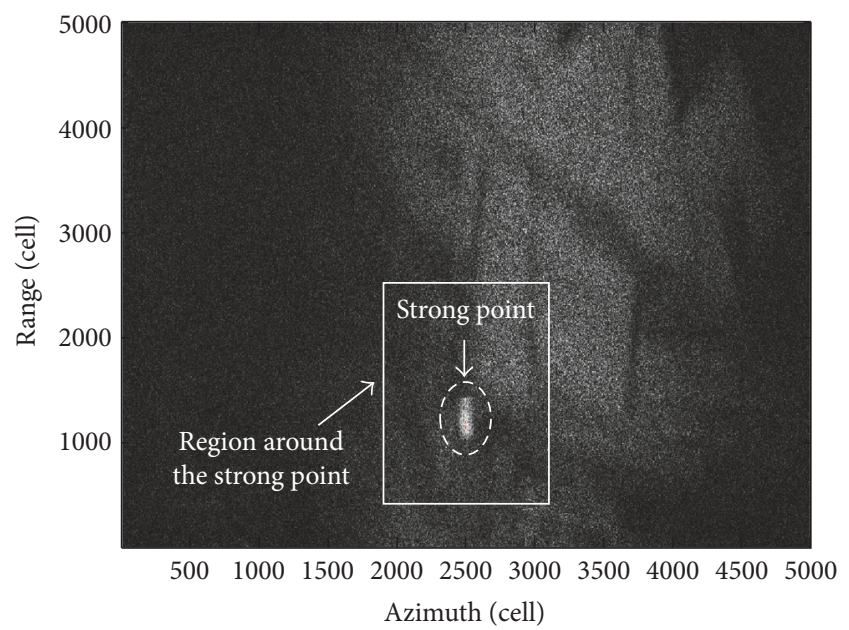

FIGURE 12: Imaging result based on conventional RCMC (bistatic radar). The defocusing of the image is much more serious than that in the monostatic SAR due to the larger RCM.

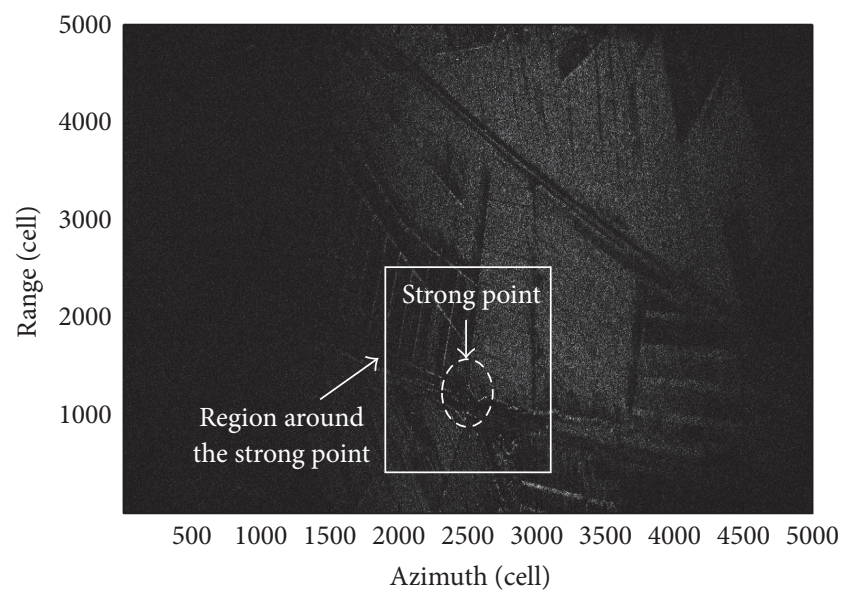

FIGURE 13: Imaging result with CF-RCMC (bistatic radar). By using the RCMC method proposed in this paper, the image is well focused.

greatly improves the focusing quality of the imaging for the bistatic SAR. Particularly, the focusing performance in the area around the selected strong point target is excellent.

4.4. Discussion. In this paper, the proposed CF-RCMC method has been demonstrated to be more effective than the conventional RCMC technique. It is necessary to note that there is an important difference between the existing RCMC and our proposed CF based method. As we know, the traditional RCMC methods generally choose the nearest slant range as references, further derive the migration momentum of the reference points mathematically, and then move the distance curves to the nearest slope distance in time domain or frequency domain [20,21]. Although these methods are useful in several scenarios, they do not take into account the parametric uncertainty of the system in actual flight cases, which lead to a large error between the migration equation and the actual migration curve. Additionally, another 
disadvantage is that, under different SAR modes such as bistatic or monostatic, the existing RCMC may not be generalized in applications.

Comparatively, the proposed CF-RCMC in this paper is motivated in a different view. Generally, from the magnitude trajectory of the actual echo strong points, the CF-RCMC aims at adjusting the calibration parameters such that the distance curve can coincide with the trajectory of the strong points of the echo. Then, the migration correction is implemented. The advantages of the proposed method are that, on the one hand, it is robust to the uncertainty of the system parameters and, on the other hand, it can also be adapted to different SAR modes (i.e., monostatic and bistatic).

However, in fact, no matter which RCMC methods are used, the correction results of nonreference points cannot be statistically assessed, and therefore we have to evaluate the effectiveness of RCMC methods from the imaging result after azimuth compression. From our observation in experiment, the regression distance curve coincides with the trajectory of the strengths of the echo completely; that is, the regression accuracy for the strong points of the echo is $100 \%$. However, the regression accuracy of other points (nonstrong points) cannot be estimated due to the fact that the trajectory in echo cannot be observed. In the future, machine learning based methods $[27,28]$ can be further considered for handling the issues.

\section{Conclusions}

The correction for range cell migration caused by the motion of the radar relative to the target plays an important role in the RD algorithm in SAR. In this paper, a novel CF-RCMC method based on the polynomial curve fitting under the least squares criterion is proposed. This method is applicable not only to the monostatic SAR but also to the bistatic SAR and even to any kind of SAR configuration. The high flexibility of this method is significantly important for the simplification of CF-RCMC. The experimental results of both the monostatic SAR and the bistatic SAR confirm the effectiveness of the proposed CF-RCMC method.

\section{Conflicts of Interest}

The authors declare that there are no conflicts of interest regarding the publication of this paper.

\section{Acknowledgments}

This work was supported by Fundamental Research Funds for the Central Universities (no. 106112017CDJQJ168819).

\section{References}

[1] C. W. Sherwin, J. P. Ruina, and R. D. Rawcliffe, "Some Early Developments in Synthetic Aperture Radar Systems," IRE Transactions on Military Electronics, vol. 6, no. 2, pp. 111-115, 1962.

[2] Y. T. Liu, Radar Imaging Technology, Harbin Institute of Technology Press, 1999.
[3] M. Weib, J. H. G. Ender, and C. H. Gierull, "Foreword to the special issue on scientific and technological progress of synthetic aperture radar (SAR)," IEEE Transactions on Geoscience and Remote Sensing, vol. 51, no. 8, pp. 4363-4365, 2013.

[4] N. Pierdicca, P. Castracane, and L. Pulvirenti, "Inversion of electromagnetic models for bare soil parameter estimation from multifrequency polarimetric SAR data," Sensors, vol. 8, no. 12, pp. 8181-8200, 2008.

[5] X. Huang, B. Huang, and H. Li, "A fast level set method for synthetic aperture radar ocean image segmentation," Sensors, vol. 9, no. 2, pp. 814-829, 2009.

[6] H. Li, Y. He, and W. Wang, "Improving ship detection with polarimetric SAR based on convolution between copolarization channels," Sensors, vol. 9, no. 2, pp. 1221-1236, 2009.

[7] X. Zhou, N.-B. Chang, and S. Li, "Applications of SAR interferometry in earth and environmental science research," Sensors, vol. 9, no. 3, pp. 1876-1912, 2009.

[8] X. Zhou, G. Wei, S. Wu, and D. Wang, “Three-dimensional ISAR imaging method for high-speed targets in short-range using impulse radar based on SIMO array," Sensors (Switzerland), vol. 16 , no. 3 , article $364,2016$.

[9] I. Cumming and F. Wong, Digital Processing of Synthetic Aperture Radar Data: Algorithms and Implementation, Artech House, 2005.

[10] J. J. M. De Wit, A. Meta, and P. Hoogeboom, "Modified rangedoppler processing for FM-CW synthetic aperture radar," IEEE Geoscience and Remote Sensing Letters, vol. 3, no. 1, pp. 83-87, 2006.

[11] E. C. Zaugg and D. G. Long, "Generalized frequency-domain SAR processing," IEEE Transactions on Geoscience and Remote Sensing, vol. 47, no. 11, pp. 3761-3773, 2009.

[12] H.-S. Shin and J.-T. Lim, "Omega-k algorithm for airborne spatial invariant bistatic spotlight SAR imaging," IEEE Transactions on Geoscience and Remote Sensing, vol. 47, no. 1, pp. 238-250, 2009.

[13] S. Jun, M. Long, and Z. Xiaoling, "Streaming BP for nonlinear motion compensation SAR imaging based on GPU," IEEE Journal of Selected Topics in Applied Earth Observations and Remote Sensing, vol. 6, no. 4, pp. 2035-2050, 2013.

[14] C. Wu, "A digital system to produce imagery from SAR data," in Proceedings of the Systems Design Driven by Sensors, pp. 76-968, 1976.

[15] R. Lanari and G. Fornaro, "A short discussion on the exact compensation of the SAR range-dependent range cell migration effect," IEEE Transactions on Geoscience and Remote Sensing, vol. 35, no. 6, pp. 1446-1452, 1997.

[16] J. C. Curlander and R. N. McDonough, Synthetic Aperture Radar: Systems and Signal Processing, Wiley, Hoboken, NJ, USA, 1991.

[17] R. Bamler, "A comparison of range-Doppler and wavenumber domain SAR focusing algorithms," IEEE Transactions on Geoscience and Remote Sensing, vol. 30, no. 4, pp. 706-713, 1992.

[18] C. Wu, K. Y. Liu, and M. Y. Jin, "Modeling and a correlation algorithm for spaceborne SAR signals," IEEE Transactions on Aerospace and Electronic Systems, vol. 18, no. 5, pp. 563-575, 1982.

[19] W. Junfeng and L. Xingzhao, "Automatic range-migration correction in sar imaging," in Proceedings of the 2008 IEEE International Geoscience and Remote Sensing Symposium Proceedings, pp. IV1257-IV1260, usa, July 2008. 
[20] T. Zeng, W. Yang, Z. Ding, and L. Liu, "Advanced range migration algorithm for ultra-high resolution spaceborne synthetic aperture radar," IET Radar, Sonar and Navigation, vol. 7, no. 7, pp. 764-772, 2013.

[21] W. Wang, W. Wu, W. Su, R. Zhan, and J. Zhang, "High squint mode SAR imaging using modified RD algorithm," in Proceedings of the 2013 IEEE China Summit and International Conference on Signal and Information Processing (ChinaSIP), pp. 589-592, July 2013.

[22] S.-X. Zhang, M.-D. Xing, X.-G. Xia, L. Zhang, R. Guo, and Z. Bao, "Focus improvement of high-squint SAR based on azimuth dependence of quadratic range cell migration correction," IEEE Geoscience and Remote Sensing Letters, vol. 10, no. 1, pp. 150-154, 2013.

[23] G. Garza and Z. Qiao, "Resolution analysis of bistatic SAR," in Proceedings of the Radar Sensor Technology XV, usa, April 2011.

[24] J. Lopez, G. Garza, and Z. Qiao, "Cross-range imaging of SAR data," Pacific Journal of Applied Mathematics, vol. 2, 65 pages, 2009.

[25] L. Zhang, H.-L. Li, Z.-J. Qiao, M.-D. Xing, and Z. Bao, "Integrating autofocus techniques with fast factorized backprojection for high-resolution spotlight SAR imaging," IEEE Geoscience and Remote Sensing Letters, vol. 10, no. 6, pp. 13941398, 2013.

[26] L. Grama and C. Rusu, "Phase approximation by divide-andconquer piecewise linear fitting of gain," in Proceedings of the 2009 International Symposium on Signals, Circuits and Systems, ISSCS 2009, rou, July 2009.

[27] L. Zhang and D. Zhang, "Robust Visual Knowledge Transfer via Extreme Learning Machine-Based Domain Adaptation," IEEE Transactions on Image Processing, vol. 25, no. 10, pp. 4959-4973, 2016.

[28] L. Zhang, W. Zuo, and D. Zhang, "LSDT: Latent Sparse Domain Transfer Learning for Visual Adaptation," IEEE Transactions on Image Processing, vol. 25, no. 3, pp. 1177-1191, 2016. 


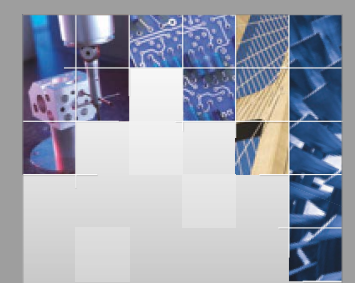

\section{Enfincering}
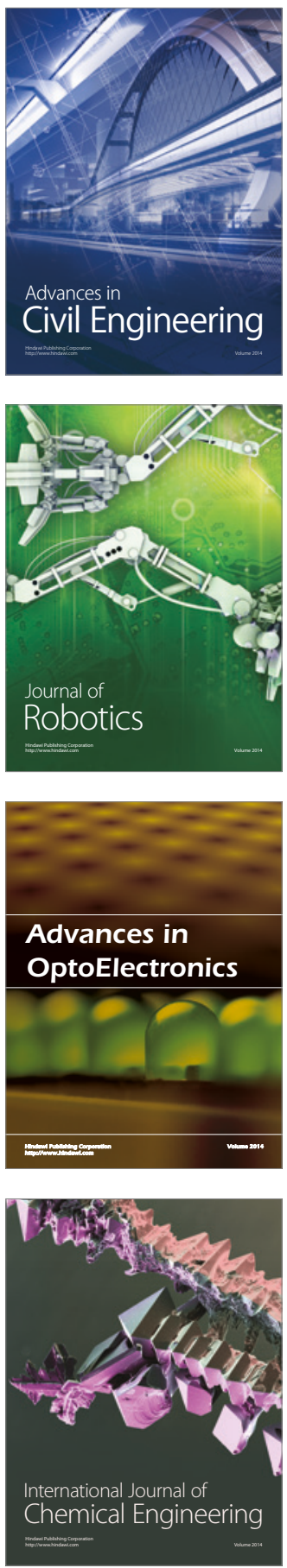

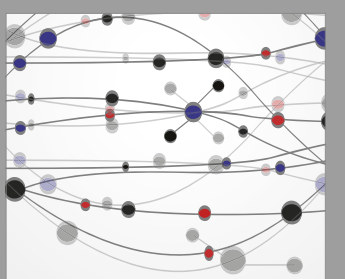

The Scientific World Journal

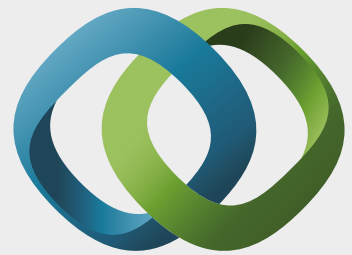

\section{Hindawi}

Submit your manuscripts at

https://www.hindawi.com
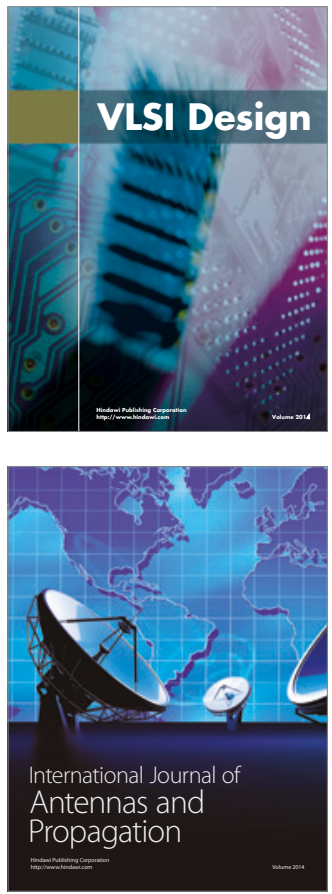

\section{Rotating}

Machinery
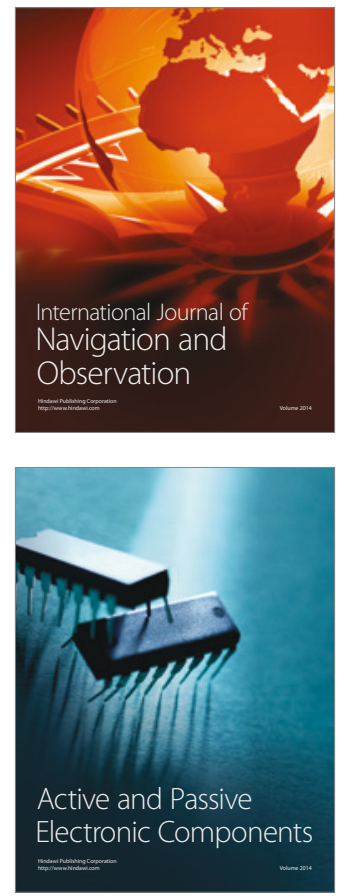
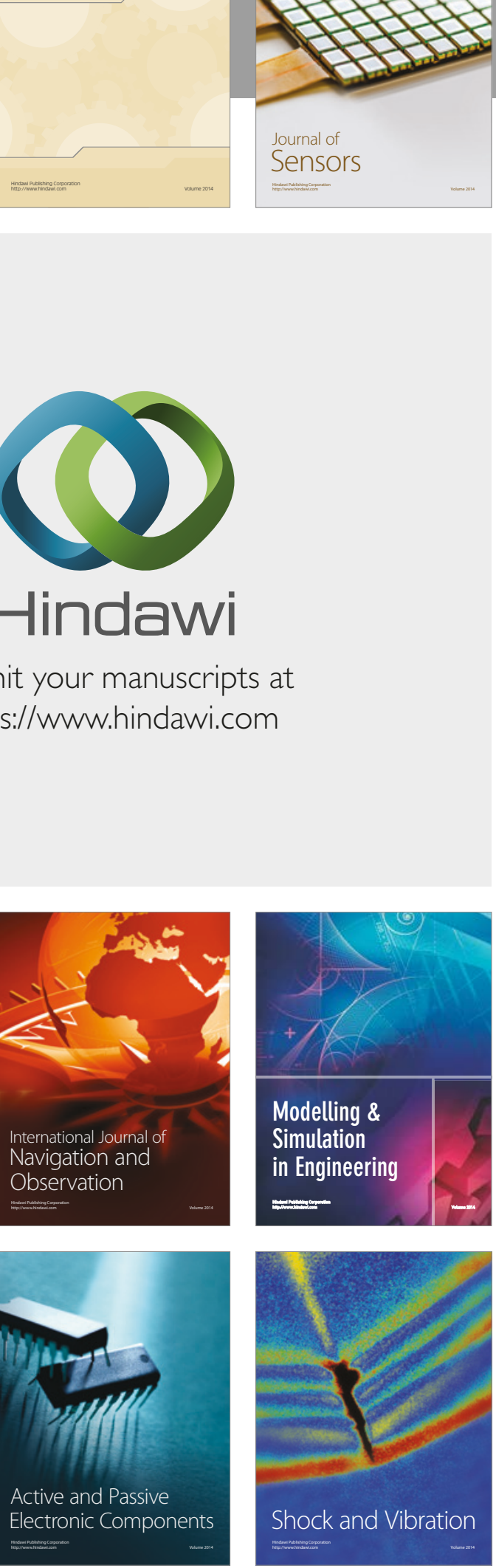
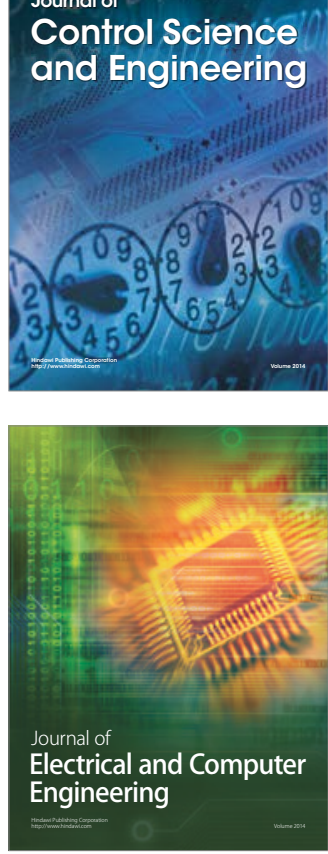

Distributed

Journal of

Control Science

and Engineering
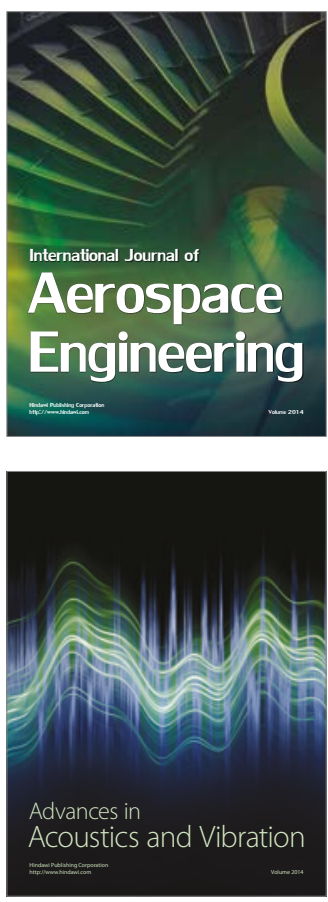

Sensor Networks 